\title{
Observation of density segregation inside migrating dunes
}

\author{
Christopher Groh, ${ }^{1}$ Ingo Rehberg, ${ }^{1}$ and Christof A. Kruelle ${ }^{1,2, *}$ \\ ${ }^{1}$ Experimentalphysik V, Universität Bayreuth, D-95440 Bayreuth, Germany \\ ${ }^{2}$ Maschinenbau und Mechatronik, Hochschule Karlsruhe-Technik und Wirtschaft, D-76133 Karlsruhe, Germany
}

(Received 10 November 2010; revised manuscript received 12 September 2011; published 9 November 2011)

\begin{abstract}
Spatiotemporal patterns in nature, such as ripples or dunes, formed by a fluid streaming over a sandy surface show complex behavior despite their simple forms. Below the surface, the granular structure of the sand particles is subject to self-organization processes, exhibiting such phenomena as reverse grading when larger particles are found on top of smaller ones. Here we report results of an experimental investigation with downscaled model dunes revealing that, if the particles differ not in size but in density, the heavier particles, surprisingly, accumulate in the central core close to the top of the dune. This finding contributes to the understanding of sedimentary structures found in nature and might be helpful to improve existing dating methods for desert dunes.
\end{abstract}

DOI: 10.1103/PhysRevE.84.050301

If a fluid streams over an extended area of sand, the grains will self-organize by forming complex granular structures such as ripples or dunes [1]. The dynamics of these systems is determined by the individual fate of the particles [2]. In general, agitated granular matter is known to show demixing whenever particles differ in size or density [3], and indeed size segregation is a well-known feature of ripples and dunes [4-16], as reviewed by Kleinhans [17].

In 1993 Anderson and Bunas [8] demonstrated the effect of size segregation in a migrating dune by modeling the trajectories of large and small particles with a stochastic cellular automaton. This kind of particle sorting observed in natural dunes is known to geomorphologists as "reverse grading,"where larger particles are more likely to be found in the upper parts of a dune. These coarsened crests occur only for fully developed dunes, when their lee slopes are shadowed from impacting particles. Then, the trajectory of an individual grain behind the dune's crest depends strongly on the wind velocity field and its mass. Larger, and therefore heavier, grains travel in small jumps of the order of a few grain diameters, while smaller particles are able to leap over the crest far down into the shadow zone. Consequently, smaller particles end up being buried by larger ones at the toe of a dune.

In addition, Makse [10] showed that size segregation due to different hopping lengths in the wake of a dune competes with a so-called shape segregation during transport and rolling of particles with different roughness along the dune's surface. These two different segregation mechanisms in aeolian sand ripples lead to a so-called "inverse grading climbing ripple lamination" and "cross-stratification patterns," respectively. The origin of the cross-stratification structures is reminiscent of recent experiments of avalanche segregation of mixtures of large faceted grains and small rounded grains poured in a vertical Hele-Shaw cell $[18,19]$.

If, however, the particles inside a dune differ not in size or shape but in density, the question arises as to whether a similar demixing of heavy and light grains can be found as in other granular systems [3]. Up to now, and to the best of our knowledge, this process of density segregation during dune migration has not been observed experimentally. By using a

*christof.kruelle@hs-karlsruhe.de
PACS number(s): 45.70.-n

bi-dense mixture of equally sized particles we demonstrate the counterintuitive effect that the heavier particles accumulate in the central core close to the top of the dune. This result provides a clue toward an advanced understanding of the sedimentary structures found in nature [20-22]. It might be helpful to improve existing dating methods for desert dunes $[23,24]$ and it can illuminate the origin of placers of minerals in various sediments [25].

Our experimental setup mainly consists of a narrow water flow channel, which allows the experimental investigation of downsized slices of transverse dunes [26,27]. The width of the channel amounts to $6 \mathrm{~mm}$ and the height is $6 \mathrm{~cm}$. We measure the fixed unidirectional flow velocity of $0.60 \mathrm{~m} / \mathrm{s}$ with an ultrasonic Doppler velocimeter. The corresponding Reynolds number of $\operatorname{Re}=36000$ is calculated with the height of the channel. After the flume is filled with distilled water, two different types of beads are poured into the channel. One type is made of glass with a density of $\rho_{\mathrm{g}}=2.5 \mathrm{~g} / \mathrm{cm}^{3}$. The other ones are white ceramic beads with a density of $\rho_{\mathrm{c}}=$ $6.0 \mathrm{~g} / \mathrm{cm}^{3}$. Both particle types have the same mean diameter of $0.5 \mathrm{~mm}$ in order to exclude size segregation in the form of inverse grading [4]. A camera records side views of the dunes as shown in Fig. 1. After suitable image processing one can clearly distinguish between the black background, the glass beads appearing gray, and the white ceramic beads.

For the experiment shown in Fig. 1 we choose a 50:50 by volume mixture of glass and ceramic beads. The ceramic beads are poured into the channel before the glass beads are filled in. The resulting starting configuration is shown in Fig. 1 at $t=0 \mathrm{~s}$. After $1 \mathrm{~min}$ of continuous and unidirectional water flow has elapsed, this initial configuration is inverted. The lighter glass beads have settled at the bottom of the dune while the heavier ceramic ones appear at the top. Moreover, the experiment reveals the nature of this paradox effect, which is due to three mechanisms. (i) The first one is similar to an effect known from agriculture, namely, the separation of the "wheat" from the "chaff." In our case the lighter particles are dragged by the water flow on longer flights than the heavier ones. (ii) Further downstream the glass beads are caught in the wake region due to the characteristic recirculating flow [28] and form an elongated nose as indicated in Fig. 1. (iii) They provide the carpet for the dune to roll over. This explains why the lighter particles always end up at the bottom. 


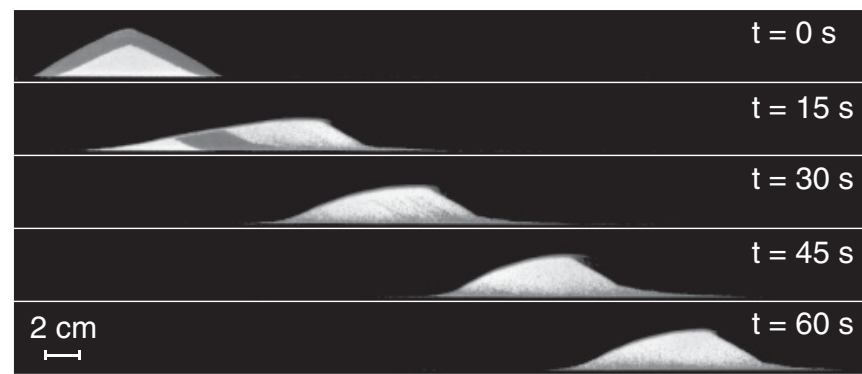

FIG. 1. Temporal evolution of a bi-dense dune: Five timesequenced snapshots showing side views of a developing dune slice composed of glass beads, appearing gray, and white ceramic beads. The direction of the steady water flow is from left to right.

To test the universality of the observed segregation effect we prepared two other initial starting configurations, namely, the inverse configuration with the lighter particles at the bottom [see Fig. 2(b)], and a mixed configuration. The mixed configuration is the attempt to achieve a homogeneous mixture of the two types of beads, which succeeds in a beaker, but fails during filling this melange into the channel. The different densities lead to a configuration as shown in Fig. 2(c), where the heavier beads sediment preferably in the center. The overall mass at each of the three experiments remains constant and amounts to $m=22.1 \mathrm{~g}$, as the volume fraction of ceramic beads is $\varphi=50 \%$.

Making use of the fact that the three initial configurations achieve one common steady state, we averaged the images during the last $5 \mathrm{~s}$ of each measurement. The result is shown in Fig. 2(d) and represents the attractor. The color code indicates
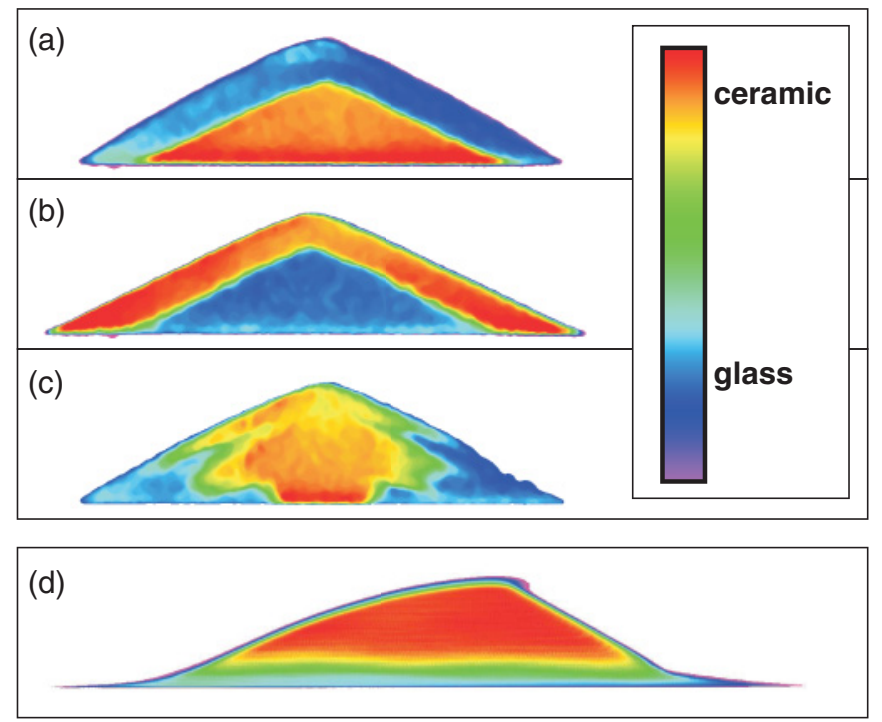

FIG. 2. (Color) Initial preparation and final segregated state for three different starting configurations: (a) heavy particles on bottom, (b) heavy particles on top, and (c) mixed. (d) shows the mass distribution within the common steady-state dune slice. The false color code corresponds to the density of the particles, i.e., red represents heavy material and blue represents light material. A median filter smooths the rendering of the beads in (a)-(c). The height of each panel corresponds to $4.3 \mathrm{~mm}$ in the experiment.

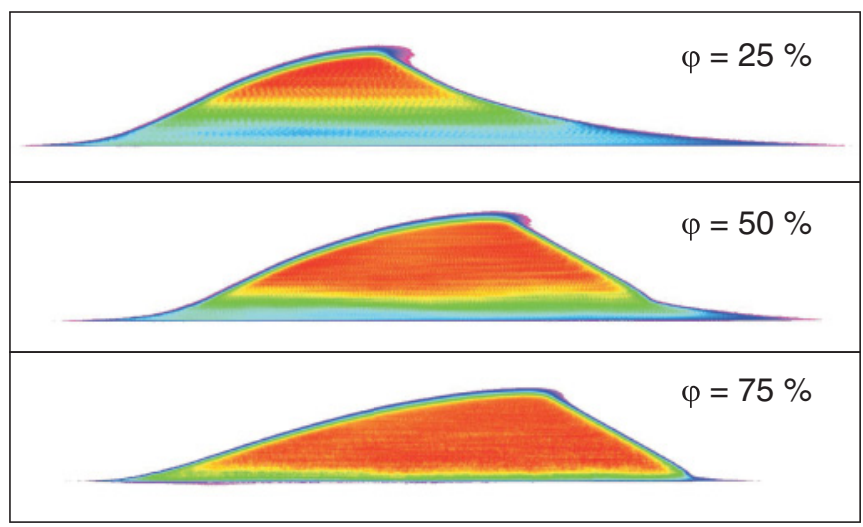

FIG. 3. (Color) Final segregated states for three bi-dense mixtures with different volume fractions $\varphi$ of ceramic beads. The color code corresponds to the one in Fig. 2. The height of each panel corresponds to $4.3 \mathrm{~mm}$ in the experiment.

the density distribution of the particles, which is similar to the one shown in Fig. 1 at $t=60 \mathrm{~s}$.

Another option to test the robustness of the observed segregation effect is the change of the volume fraction $\varphi$ of dense ceramic beads. This means that the total size of the dune is kept constant, while the percentage of heavy beads is varied. Figure 3 shows that the heavy beads always end up on top of the dune. However, the contours of the dunes differ slightly. At $\varphi=25 \%$ the downstream nose is more pronounced and the slipface formed by the ceramic beads is smaller.

To characterize the dynamical behavior, Fig. 4 shows the dune velocity $v$ as a function of its mass. It is known that in the scaling limit the velocity will scale with the square root of the mass, if the dunes consist of only one particle species [29]. In particular, it was demonstrated that this scaling holds for at least one and a half decades and for three different Reynolds numbers [26,27]. The data shown in Fig. 4 for pure glass and pure ceramic dunes, respectively, are in agreement with that fact, as indicated by the dashed black lines, which are fits to $v \sim m^{-0.5}$.

However, for dunes consisting of more particle species of different density, we expect a scaling according to $v \sim \rho_{\text {mean }}^{-1.5}$, with the mean density of the mixture given as $\rho_{\text {mean }}=\rho_{\mathrm{g}}+$ $\varphi\left(\rho_{\mathrm{c}}-\rho_{\mathrm{g}}\right)$ (see the inset of Fig. 4). This exponent can be explained with the finding of Bagnold [1] that the migration velocity of a steady-state dune is inversely proportional to the density $\rho_{\text {mean }}$ of its sand. Combining both ideas we get $v \sim \rho_{\text {mean }}^{-1} m^{-0.5}$, which finally leads to

$$
v \sim m^{-1.5} \text { or } \quad v \sim \rho_{\text {mean }}^{-1.5},
$$

respectively, under the restriction of a constant volume. The corresponding fits are shown as red lines in Fig. 4.

In addition, Fig. 5 shows the results of an analysis of the same data as displayed in Fig. 4. Here, the dune velocities for a fixed mass $m=22.1 \mathrm{~g}$ (see Fig. 1) have been derived from the prefactors of the scaling laws for the migration velocity $v$ versus dune mass $m$. It can be clearly seen that, for a constant mass, the migration velocity scales, as suggested by Bagnold [1] in Chap. 13, inversely proportional to the average density of the dune's particles and thus linearly with the volume of the dune. 

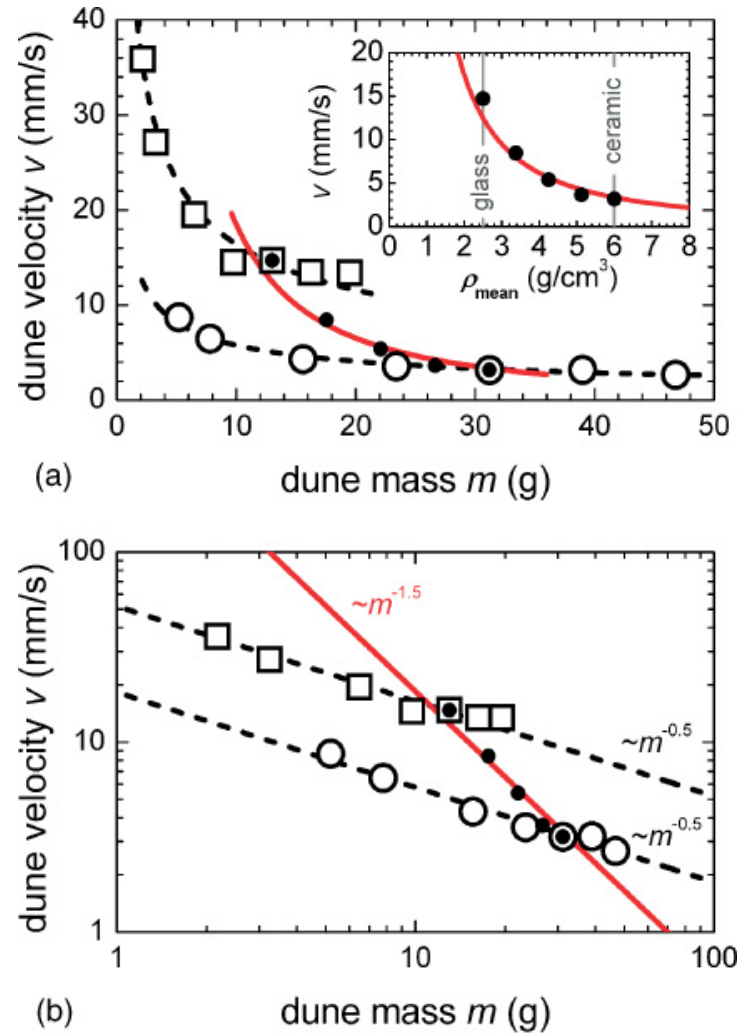

FIG. 4. (Color) Linear (a) and double logarithmic plot (b) of the migration velocity vs dune mass for dunes composed entirely of glass beads (squares) or ceramic beads (circles). For bi-dense dunes (full disks) the volume is kept constant, but the volume fraction $\varphi$ of ceramic beads is varied, which leads to different dune masses. The dashed black lines are power-law fits with an exponent of $-1 / 2$, whereas the solid red lines indicate power-law fits with an exponent of $-3 / 2$. In the inset the dune velocity $v$ of bi-dense dunes is replotted as a function of the mean particle density $\rho_{\text {mean }}$.

In conclusion, our measurement technique allows to study segregation phenomena both outside and inside migrating dunes with high spatial and temporal resolution. This method can even be used for tracking all individual particles as demonstrated in Ref. [2], which revealed the inner dynamics of a barchan dune slice. Our experiment presents the counterintuitive effect that in bi-dense packings the heavier grains accumulate at the top of migrating dunes while lighter particles are buried at the bottom. If the particles' densities were distributed continuously, results comparable to the inverse grading of polydisperse packings would be expected.

As a side effect, we show that the migration velocity of bi-dense dunes scales with the mean density of the grains as a power-law function with an exponent of $-3 / 2$. This experimental observation raises the question as to whether

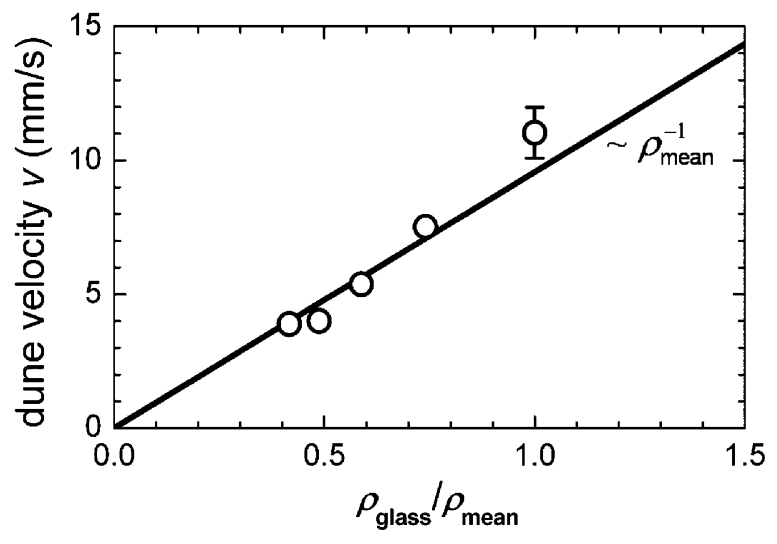

FIG. 5. Migration velocity $v$ vs normalized inverse average density $\rho_{\text {glass }} / \rho_{\text {mean }}$ for dunes composed of glass beads and ceramic beads. Here the mass $m=22.1 \mathrm{~g}$ is kept constant, but the volume fraction $\varphi$ of ceramic beads is varied, which leads to different dune volumes. The solid line is a linear fit to the data.

the migration velocity only depends on $\rho_{\text {mean }}$ or whether the distribution of densities also plays a role.

Another issue is the case of transverse dunes. For tackling this task experiments with a completely covered floor are needed. There, the dynamics of a rippled surface developing from an initially flattened bottom has been studied to a great extent using a single-particle species. For bi-dense packings it is expected that similar segregation phenomena are observed as for single barchan dunes, with the additional feature that lighter particles leaving the lee side of the dune will eventually also cover the windward side of its downstream neighbor.

For further examining the underlying mechanisms of the segregation phenomena observed so far, one has to distinguish between size, shape, and density segregation by examining differences in suspended flight behavior between larger and smaller particles and denser and less dense particles. Based on our experimental findings, it might be speculated that the particle's mass is the most relevant parameter. Here the properties of the driving fluid also play a role: e.g., for lower Reynolds numbers, different mechanisms of entrainment of particles into the fluid and their successive redeposition on the dune's surface might result in a different outcome of the segregation patterns.

Loosely speaking, these insights into the sedimentology of dunes composed of different types of sand have the implication that in a ripple or dune mixed of gold and sand, the gold nuggets are likely to be found at the top, close to the surface at the crest.

Financial support by the German Science Foundation within Forschergruppe 608 "Nichtlineare Dynamik komplexer Kontinua" through Grant No. Kr1877/3-1 is gratefully acknowledged.
[1] R. A. Bagnold, The Physics of Blown Sand and Desert Dunes (Methuen, London, 1941).

[2] C. Groh, I. Rehberg, and C. A. Kruelle, New J. Phys. 12, 063025 (2010).

[3] A. Kudrolli, Rep. Prog. Phys. 67, 209 (2004).
[4] R. E. Hunter, Sedimentology 24, 361 (1977).

[5] R. D. Sarre and C. C. Chancey, Sedimentology 37, 357 (1990).

[6] H. Tsoar, Geogr. Res. Forum 10, 37 (1990).

[7] S. G. Fryberger, P. Hesp, and K. Hastings, Sedimentology 39, 319 (1992). 
[8] R. S. Anderson and K. L. Bunas, Nature (London) 365, 740 (1993).

[9] P. Y. Julien, Y. Lan, and G. Berthault, Bull. Soc. géol. France 5, 649 (1993).

[10] H. A. Makse, Eur. Phys. J. E 1, 127 (2000).

[11] H. Caps and N. Vandewalle, Physica A 313, 357 (2002).

[12] S. A. Wilson, J. R. Zimbelman, and S. H. Williams, Lunar Planet. Sci. XXXIV, 1862 (2003).

[13] W. G. Nickling, C. M. Neuman, and N. Lancaster, Sedimentology 49, 191 (2004).

[14] G. Rousseaux, H. Caps, and J.-E. Wesfreid, Eur. Phys. J. E 13, 213 (2004).

[15] D. J. Jerolmack, D. Mohrig, J. P. Grotzinger, D. A. Fike, and W. A. Watters, Mars. J. Geophys. Res. 111, E12S02 (2006).

[16] E. Manukyan and L. Prigozhin, Phys. Rev. E 79, 031303 (2009).

[17] M. G. Kleinhans, Earth Sci. Rev. 65, 75 (2004).

[18] H. A. Makse et al., Nature (London) 386, 379 (1997).
[19] P. Cizeau, H. A. Makse, and H. E. Stanley, Phys. Rev. E 59, 4408 (1999).

[20] J. R. L. Allen, Sedimentary Structures: Their Character and Physical Basis (Elsevier, Amsterdam, 1982).

[21] J. D. Collinson and D. B. Thompson, Sedimentary Structures (George Allen \& Unwin, London, 1982).

[22] C. S. Bristow, S. D. Balley, and N. Lancaster, Nature (London) 406, 56 (2000).

[23] M. Schuster et al., Science 311, 821 (2006).

[24] J. Sun and T. Liu, Science 312, 1621 (2006).

[25] J. Saxton, P. Fralick, U. Panu, and K. Wallace, Econ. Geol. 103, 1657 (2008).

[26] C. Groh, A. Wierschem, N. Aksel, I. Rehberg, and C. A. Kruelle, Phys. Rev. E 78, 021304 (2008).

[27] C. Groh, A. Wierschem, N. Aksel, I. Rehberg, and C. A. Kruelle, Phys. Rev. E 79, 019903(E) (2009).

[28] H. Ayrton, Proc. R. Soc. London A 84, 285 (1910).

[29] B. Andreotti, P. Claudin, and S. Douady, Eur. Phys. J. B 28, 341 (2002). 